\title{
Memory reconsolidation mediates the updating of hippocampal memory content
}

\author{
Jonathan L. C. Lee* \\ School of Psychology, University of Birmingham, Birmingham, UK
}

\section{Edited by:}

Jacek Debiec, New York University, USA

\section{Reviewed by:}

Valerie Doyere, National Centre for

Scientific Research, France

Jacek Debiec, New York University, USA

\section{*Correspondence:}

Jonathan L. C. Lee, School of

Psychology, University of Birmingham

Edgbaston, Birmingham B15 2TT, UK.

e-mail:j.l.c.lee@bham.ac.uk
The retrieval or reactivation of a memory places it into a labile state, requiring a process of reconsolidation to restabilize it. This retrieval-induced plasticity is a potential mechanism for the modification of the existing memory. Following previous data supportive of a functional role for memory reconsolidation in the modification of memory strength, here I show that hippocampal memory reconsolidation also supports the updating of contextual memory content. Using a procedure that separates the learning of pure context from footshock-motivated contextual fear learning, I demonstrate doubly dissociable hippocampal mechanisms of initial context learning and subsequent updating of the neutral contextual representation to incorporate the footshock. Contextual memory consolidation was dependent upon BDNF expression in the dorsal hippocampus, whereas the footshock modification of the contextual representation required the expression of Zif268. These mechanisms match those previously shown to be selectively involved in hippocampal memory consolidation and reconsolidation, respectively. Moreover, memory reactivation is a necessary step in modifying memory content, as inhibition of hippocampal synaptic protein degradation also prevented the footshock-mediated memory modification. Finally, dorsal hippocampal knockdown of Zif268 impaired the reconsolidation of the pure contextual memory only under conditions of weak context memory training, as well as failing to disrupt contextual freezing when a strong contextual fear memory is reactivated by further conditioning. Therefore, an adaptive function of the reactivation and reconsolidation process is to enable the updating of memory content.

Keywords: reconsolidation, hippocampus, fear conditioning, contextual learning, Zif268, BDNF, memory

\section{INTRODUCTION}

Reactivation-dependent or cue-dependent amnesia is consistently observed in many behavioral paradigms, and is commonly interpreted as reflecting the disruption of a reconsolidation process that serves to restabilize reactivated memories (Nader and Hardt, 2009). However, reconsolidation may serve a wider purpose than simply restabilizing memories that have been destabilized through retrieval. Thus it has been suggested that the retrieval-induced plasticity that characterizes the reconsolidation process is ideally placed to mediate the modification of existing memories (Sara, 2000; Dudai, 2006; Hupbach et al., 2007; Lee, 2009; Winters et al., 2009).

Hippocampal contextual fear memories are an ideal substrate for the analysis of memory reconsolidation function, as there are doubly dissociable cellular mechanisms of consolidation and reconsolidation (Lee et al., 2004). Therefore, it is possible selectively to disrupt the reconsolidation process under conditions of memory modification. Using such an approach, I have previously established that hippocampal memory reconsolidation is a primary mechanism by which contextual fear memories are strengthened through further learning (Lee, 2008). However, it remains unclear whether such an adaptive function can be extended to the modification of memory content.

Data from human episodic memory studies suggest that memory retrieval is sufficient to enable the incorporation of new information into a memory for a list of items (Hupbach et al., 2007;
Forcato et al., 2010). While this is consistent with a functional role for reconsolidation in the memory modification process, it does not necessarily indicate that the episodic memory is reactivated and reconsolidated to form a modified trace, rather than memory retrieval enabling the integration of two complementary or competing traces. Therefore, a cellular approach is needed to substantiate the selective contribution of reconsolidation mechanisms to the modification of memory content. An initial study in rodent inhibitory avoidance memories, also capitalizing upon doubly dissociable mechanisms of memory consolidation and reconsolidation (Taubenfeld et al., 2001; Milekic et al., 2007), concluded that reconsolidation was not required to link new information to a retrieval memory (Tronel et al., 2005). However, as noted by Tronson and Taylor (2007), the second-order conditioning procedure used to assess the linking of new information likely reflects a new independent association, rather than an updated existing memory.

In the present study, I investigated the functional role of a selective dorsal hippocampal reconsolidation mechanism (the expression of the immediate-early gene Zif268) in the updating of hippocampal memory content. In the context pre-exposure facilitation effect (CPFE) paradigm, rats are first exposed to a novel context alone. Upon subsequent return to the now familiar context, the rats receive an immediate footshock, resulting in an association between the footshock and the retrieved memory of the context that is expressed as increased conditioned freezing behavior at a later context exposure test (Biedenkapp and Rudy, 2004). Within such a 
procedure, it has been suggested that the contribution of the hippocampus to contextual fear conditioning is simply to encode the spatial representation of the context (Fanselow, 2000; Rudy et al., 2002), which is likely associated with the footshock in the amygdala (Fendt and Fanselow, 1999; Matus-Amat et al., 2007). However, there is emerging evidence indicating that the hippocampus also plays a role in processing the context-shock association (Frohardt et al., 2000; Chang et al., 2008). Therefore, in the CPFE paradigm, it is possible that the hippocampal memory is modified from a neutral contextual representation to a contextual fear memory incorporating the footshock, and so this footshock-updating of the contextual memory may depend upon reconsolidation mechanisms.

It is of note that Biedenkapp and Rudy (2004) have previously found no evidence that the contextual memory representation undergoes reconsolidation, at least when reactivated by re-exposure to the context alone. If the updating of an existing memory trace to incorporate new information is a critical trigger for memory reconsolidation, exposure to the familiar context alone would not be expected to engage reconsolidation. Instead, the presentation of new information in the form of the novel and salient footshock should trigger updating, and hence reconsolidation, processes. The aforementioned doubly dissociable cellular mechanisms of hippocampal memory consolidation and reconsolidation lead to the following predictions. The acquisition of the contextual representation should be dependent upon the consolidation-specific expression of BDNF, whereas updating with the novel footshock information should selectively recruit the reconsolidation-related gene Zif268. Moreover, the involvement of reconsolidation in memory updating can be further substantiated by demonstrating the functional necessity for memory destabilization, a process that is dependent upon protein degradation and is inhibited by the proteasome inhibitor $\beta$-lac.

\section{MATERIALS AND METHODS SUBJECTS}

The subjects were 140 experimentally naïve adult male Lister Hooded rats, weighing 280-320 g at the start of the experiment. They were housed in groups of 4 , in a holding room maintained at $21^{\circ} \mathrm{C}$ on a normal light cycle $(12 \mathrm{~h}$ light: $12 \mathrm{~h}$ dark; lights on at 07:00). Food and water were freely available throughout the experiment, except during the testing sessions. All procedures were conducted in accordance with the United Kingdom 1986 Animals (Scientific Procedures) Act (Project License PPL 40/3205).

\section{SURGICAL AND INFUSION PROCEDURES}

One hundred eight rats were anesthetized using isoflurane, and were implanted with stainless steel guide cannulae (22 gauge, $11 \mathrm{~mm}$ ) aimed at the dorsal hippocampus (AP $-3.5 \mathrm{~mm}, \mathrm{~L} \pm 2.5 \mathrm{~mm}, \mathrm{~V}$ $-1.5 \mathrm{~mm}$ relative to bregma). The rats were administered with peri-operative buprenorphine analgesia and stainless steel stylets were inserted into the guide cannulae to maintain patency during recovery. A minimum of 7 days was allowed before behavioral training and testing began. At the end of the experiment, rats were perfused, and their brains were sectioned and stained to confirm cannula placements as previously described (Lee et al., 2004).

For 2 days before training began, the rats were habituated to the infusion procedure using sterile PBS vehicle. Infusions were carried out using a syringe pump (Harvard Apparatus), connected to injectors (28 gauge, projecting $2 \mathrm{~mm}$ beyond the guide cannulae) by polyethylene tubing. Infusions $(1.0 \mu \mathrm{l} /$ side $)$ were begun immediately after insertion of the injectors and were carried out over $120 \mathrm{~s}$. One minute waiting time after completion of the infusion was allowed for diffusion away from the injector tips before the injectors were withdrawn. Oligodeoxynucleotides (Alta Bioscience, UK) were PAGE-purified phosphorothioate end-capped 18-mer sequences, resuspended in sterile PBS to concentrations as used previously (Lee et al., 2004): Zif268 ASO (2 nmol/ $\mu \mathrm{l}), 5^{\prime}$-GGT AGT TGT CCA

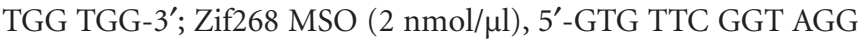
GTG TCA-3'; BDNF ASO $(1 \mathrm{nmol} / \mu \mathrm{l}), 5^{\prime}$-TCT TCC CCT TTT AAT GGT-3'; BDNF MSO (1 nmol/ $\mu \mathrm{l}), 5^{\prime}$-ATA CTT TCT GTT CTT GCC-3'. The proteasome inhibitor Clasto-Lactacystine- $\beta$-lactone $(\beta$-lac; $32 \mathrm{ng} / \mu \mathrm{l})$ was dissolved in $2 \% \mathrm{DMSO}$ in $1 \mathrm{M} \mathrm{HCl}$ diluted in PBS and adjusted to $\mathrm{pH} 7.0-7.4$ with $\mathrm{NaOH}$.

\section{BEHAVIORAL APPARATUS}

All behavioral training and testing took place in four operant chambers (MedAssociates, Vermont), each measuring $25 \mathrm{~cm} \times 32 \mathrm{~cm} \times 25.5 \mathrm{~cm}$, and housed within a sound-attenuating chamber. Three sides of the operant chambers were constructed of steel and both the ceiling and front wall were pespex, the latter also serving as a door. On the front and rear walls were located several modules (retractable levers, LED stimulus lights, food magazine, and auditory stimulus generators). However, the only module employed during these experiments was a single LED houselight, located in the top center of the rear wall of each chamber. The grid floors consisted of 19 stainless steel rods $(4.8 \mathrm{~mm}$ diameter; $1.6 \mathrm{~mm}$ center-to-center), connected to a shock generator and scrambler (MedAssociates). Below the grid floor was a removable tray. Mounted on the ceiling of the operant chamber, but within the sound-attenuating chamber, was a video camera (Viewpoint Life Sciences, France).

\section{BEHAVIORAL PROCEDURES}

The rats were subjected to a context pre-exposure and immediate shock procedure based upon Biedenkapp and Rudy (2004). In the standard procedure, rats were initially pre-exposed to the context by repeatedly transporting them in groups of 4 from the holding room to the testing room in a white bucket. The lid was kept on the bucket for transportation to the testing room, and removed for return to the holding room. Each rat was exposed to an operant chamber 6 times, the initial exposure being for $5 \mathrm{~min}$ and the remaining exposures for $40 \mathrm{~s}$ each (with the rats returned to the home cage for $40 \mathrm{~s}$ between each exposure). On the next day, the rats were transported again in the white bucket to the operant chamber, and immediately upon being placed in the chamber were administered a footshock $(2 \mathrm{~s}, 0.5 \mathrm{~mA})$ before being removed immediately and returned to the homecage. Finally, to test fear acquired to the context, the rats were placed in the operant chambers for $5 \mathrm{~min}$ and behavior was automatically scored for freezing using Videotrack software (Viewpoint Life Sciences, France). Without the context pre-exposure session, immediate footshock results in little conditioned fear to the context (the immediate shock deficit), which the context pre-exposure ameliorates (the CPFE). Oligodeoxynucleotides were infused $90 \mathrm{~min}$ prior to context preexposure or to the immediate shock session (apart from the delayed 
Zif268 group, for which the infusions were delayed until $4 \mathrm{~h}$ after the immediate shock session). Blac was infused immediately after the immediate shock session.

Several conditions were explored within this experimental procedure:

1. BDNF or Zif268 oligodeoxynucleotide infusion $90 \mathrm{~min}$ prior to the initial context exposure session to assess the contribution of hippocampal consolidation and reconsolidation mechanisms to the acquisition and consolidation of the neutral context memory.

2. BDNF or Zif268 oligodeoxynucleotide infusion 90 min prior to the immediate shock session to assess the contribution of hippocampal consolidation and reconsolidation mechanisms to the updating of the previously neutral context memory.

3. Zif268 oligodeoxynucleotide infusion $4 \mathrm{~h}$ after the immediate shock session as a delayed-infusion control.

4. $\beta$-lac infusion immediately after the immediate shock session to assess the necessity for contextual memory destabilization for memory updating.

5. Zif268 oligodeoxynucleotide infusion 90 min prior to a context memory reactivation session (2 min re-exposure to the context alone) to assess the retrieval-induced reconsolidation of the context memory.

6. Following weaker initial context pre-exposure (the training being terminated after three exposures), Zif268 oligodeoxynucleotide infusion 90 min prior to a context memory reactivation session ( $2 \mathrm{~min}$ re-exposure to the context alone) to assess the retrieval-induced reconsolidation of the weaker context memory.

7. Footshock $(0.5 \mathrm{~mA}, 0.5 \mathrm{~s})$ delivered at the end of each of the six context exposures during context pre-exposure (resulting in strong contextual fear), followed by Zif268 oligodeoxynucleotide infusion 90 min prior to an immediate shock session, to tests whether the presence of the footshock alone during immediate shock is sufficient to engage reconsolidation.

Further groups of unoperated rats were trained and, $2 \mathrm{~h}$ after the final behavioral session, the rats were terminally anesthetized and their brains extracted. A 2-mm section of the brain, from the rear bifurcation of the optic chiasm (approximately $-1.80 \mathrm{~mm}$ to $-3.80 \mathrm{~mm}$ from bregma), was taken on wet ice, and the dorsal hippocampus microdissected. The tissue was flash frozen on dry ice and stored at $-80^{\circ} \mathrm{C}$ for subsequent quantification of the levels of BDNF and Zif268 protein. Five conditions were analyzed ( $n=4$ per group): behaviorally naïve, context pre-exposure alone, immediate shock alone, context pre-exposure with subsequent immediate shock, and context pre-exposure with subsequent context memory reactivation.

\section{WESTERN BLOT ANALYSIS}

Microdissected dorsal hippocampal tissue was thawed and homogenized in lysis buffer according to previously published protocols (Ying et al., 2002). Proteins were separated on $7.5 \%$ Tris- $\mathrm{HCl}$ gels (Zif268) or $16.5 \%$ Tris-Tricine gels (BDNF) at a constant voltage of $150 \mathrm{~V}$. Transfer to nitrocellulose membranes was conducted under standard constant current conditions for $1 \mathrm{~h}$.
All subsequent procedures were conducted using the GenScript ONE-HOUR Western complete kit with TMB (GenScript, NJ, USA). Primary antibodies were used at the following concentrations: Zif268 (sc-110; Santa Cruz Biotechnology), 1:500; BDNF (sc-546; Santa Cruz Biotechnology), 1:100; $\beta$-actin (loading control; A2066; Sigma-Aldrich), 1:200. Blots were developed using TMB and were analyzed using ImageJ software according to previously published protocols (Lee et al., 2004). Briefly, loading variation was corrected by taking the mean of $\beta$-actin amounts on each blot and calculating a normalization factor for each sample that was multiplied to the raw amount of target protein. The mean was taken of the normalized protein level for the naïve control group, and the protein levels of each sample calculated as a percentage of this control mean.

\section{STATISTICAL ANALYSES}

Data are presented as mean \pm SEM percentage time freezing or $\%$ control protein levels. Data were checked for consistency, and in one case (BDNF MSO in behavioral condition (1) a subject was excluded as a statistical outlier (lying more than 2 standard deviations from the group mean). One- or two-way repeated-measures ANOVAs were performed on the data with factors treatment, condition, and test as appropriate. Tukey's test was selected for post hoc analyses, and a significance level of $p<0.05$ was selected for all analyses.

\section{RESULTS \\ WESTERN BLOT RESULTS}

While initial learning of the contextual representation engaged $B D N F$ expression in the dorsal hippocampus, the footshockupdating of the contextual representation resulted in selective upregulation of $Z$ if 268 (Figure 1). When the levels of BDNF protein were quantified, ANOVA revealed a significant overall effect $\left(F_{(4,15)}=5.093, p=0.009\right)$. Post hoc analysis $(p<0.05)$ revealed that the consolidation group had significantly higher levels of BDNF than each of the other groups. No other differences were found. Therefore, context pre-exposure engaged dorsal hippocampal consolidation mechanisms.

When the levels of Zif268 protein were quantified, ANOVA revealed a significant overall effect $\left(F_{(4,15)}=15.1, p<0.001\right)$. Post hoc analysis $(p<0.05)$ revealed that the footshock-updating group had significantly higher levels of Zif268 than each of the other groups. Moreover, the context memory reactivated group, while having lower Zif268 levels than the footshock-updating group, was also significant elevated compared to the naïve group. No other differences were found. Therefore, footshock-updating engaged dorsal hippocampal reconsolidation mechanisms. Furthermore, there was some evidence that context memory reactivation also engaged $Z$ if 268 expression, though to a lesser degree.

\section{BEHAVIORAL RESULTS \\ Histological results}

All rats had their guide cannulae placed dorsal to the dorsal hippocampus, with evidence of the injector tips having been located within the dorsal hippocampus (Figure 2). Moreover, no subjects had any extensive damage to the hippocampus or any other area of the brain. 
A

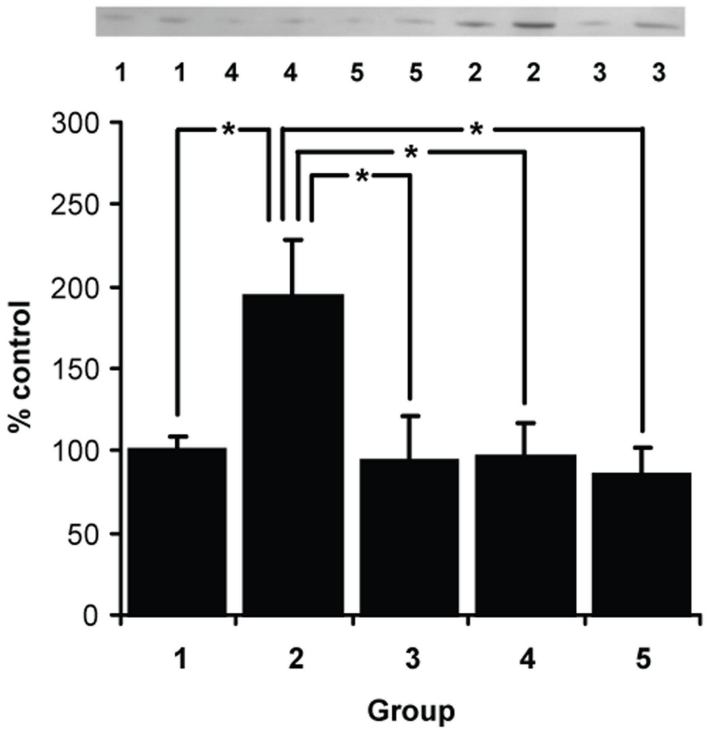

FIGURE 1 | Expression of BDNF (A) and Zif268 (B) proteins. Western blot analysis of dorsal hippocampal protein levels $2 \mathrm{~h}$ after final behavioral session in five groups. 1 - behaviorally naïve, 2 - context pre-exposure alone, 3 -immediate shock alone, 4 - context pre-exposure followed the next day by immediate shock,
B

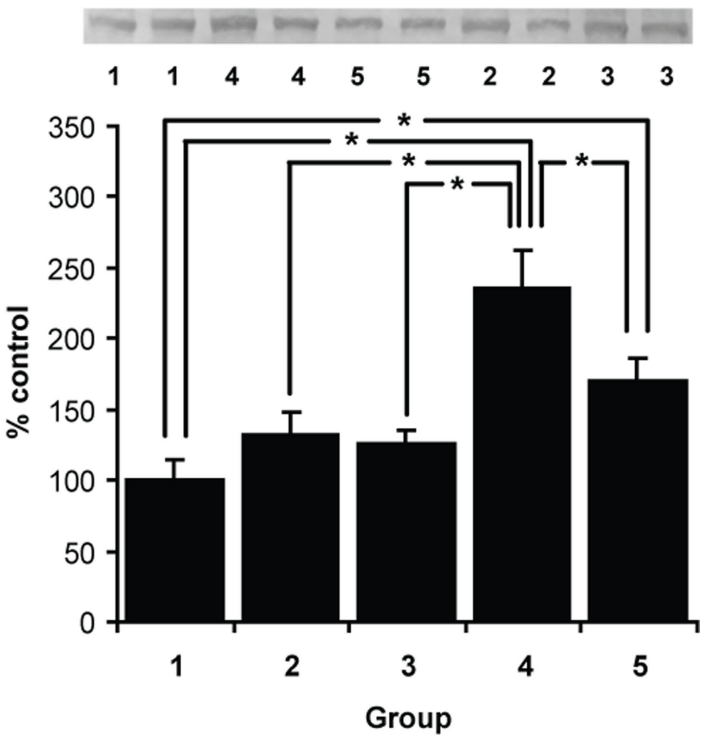

5 - context pre-exposure followed the next day by re-exposure to the context alone. Learning the context representation selectively engages BDNF, whereas associating the retrieved memory of the context with the footshock upregulates Zif268 ( $n=4$ per group). Data presented as mean + S.E.M. $\left({ }^{*} p<0.05\right)$.

\section{BDNF is required for the formation of the contextual representation}

Rats were infused into the dorsal hippocampus with BDNF or Zif268 ASO/MSO prior to context pre-exposure (Figure 3). Impairing hippocampal memory consolidation by infusion of BDNF ASO significantly impaired subsequent contextual freezing $\left(F_{(1,10)}=5.67\right.$, $p=0.039)$. In contrast, infusion of Zif268 ASO, which impairs hippocampal memory reconsolidation, had no effect relative to control missense infusions $\left(F_{(1,8)}=0.271, p=0.617\right)$. Therefore, the acquisition and consolidation of the contextual representation recruits selectively the same functional mechanism as for contextual fear memory consolidation.

\section{Zif268 and $\beta$-lac are required for footshock-updating of a contextual memory representation}

Following context pre-exposure, rats were infused into the dorsal hippocampus with Zif268 ASO/MSO, BDNF ASO/MSO, or $\beta$-lac/ PBS at the time of the immediate shock session (Figure 4A). Impairing the hippocampal reconsolidation process by infusion of Zif268 ASO significantly impaired subsequent conditioned freezing to the context, compared to Zif268 MSO-infused controls $\left(F_{(1,9)}=18.3, p=0.002\right)$. This effect was time-dependent, as it was eliminated by delaying the infusion of Zif268 to $4 \mathrm{~h}$ after the immediate shock session $\left(F_{(1,10)}=0.378, p=0.552\right)$. Moreover, prevention of memory reactivation with intra-dorsal hippocampal $\beta$-lac also impaired subsequent contextual freezing $\left(F_{(1,9)}=7.18, p=0.025\right)$. In contrast, disrupting memory consolidation mechanisms by infusing BDNF ASO had no effect relative to control missense infusions $\left(F_{(1,10)}=0.270, p=0.615\right)$.

One week after the context fear test, the Zif268 ASO/MSO and $\beta$-lac/PBS groups were again subjected to an immediate shock session, without any infusions, and subsequently tested for contextual freezing (Figure 4B). The Zif268 ASO-infused rats remained significantly impaired relative to $\mathrm{MSO}$-infused controls across both tests (Treatment $\times$ Test: $F_{(1,8)}=2.63$, $p=0.143$; Treatment: $\left.F_{(1,8)}=15.88, p=0.004\right)$, confirmed by a significant deficit in the second test when analyzed in isolation $\left(F_{(1,9)}=9.73, p=0.012\right)$. In contrast, following retraining, $\beta$-lac-infused rats were no longer impaired in comparison to PBS controls. There was a significant interaction between treatment and test $\left(F_{(1,9)}=5.89, p=0.038\right)$, with no overall effect of Treatment $\left(F_{(1,9)}=2.34, p=0.161\right)$. This was confirmed by a lack of effect of Treatment when the second test was analyzed separately $\left(F_{(1.9)}=0.191, p=0.672\right)$. The lack of contextual freezing in the Zif268 ASO group at the second test indicates that the previous treatment had not simply prevented the footshock updating of the contextual representation, but had disrupted the original representation itself. As $\beta$-lac infusions have previously been demonstrated to impair memory reactivation (Lee, 2008; Lee et al., 2008), they would be expected to prevent the destabilization of the contextual representation intact, leaving it intact and allowing the second immediate shock to condition a context-shock association. In contrast, Zif268 ASO also impaired the restabilization of the reactivated contextual memory, leaving no contextual representation to which the immediate shock could subsequently be associated. This interpretation is strengthened by the observation that a single 5 min pre-exposure to the context in unoperated rats was insufficient to overcome the immediate shock deficit (Figure 4C). There was an overall effect of condition (normal pre-exposure vs. 5 -min pre-exposure vs. no preexposure: $\left.F_{(2.9)}=23.0, p<0.001\right)$, with post hoc tests $(p<0.05)$ confirming that rats receiving the normal pre-exposure froze significantly more than rats in the other two conditions, the latter 


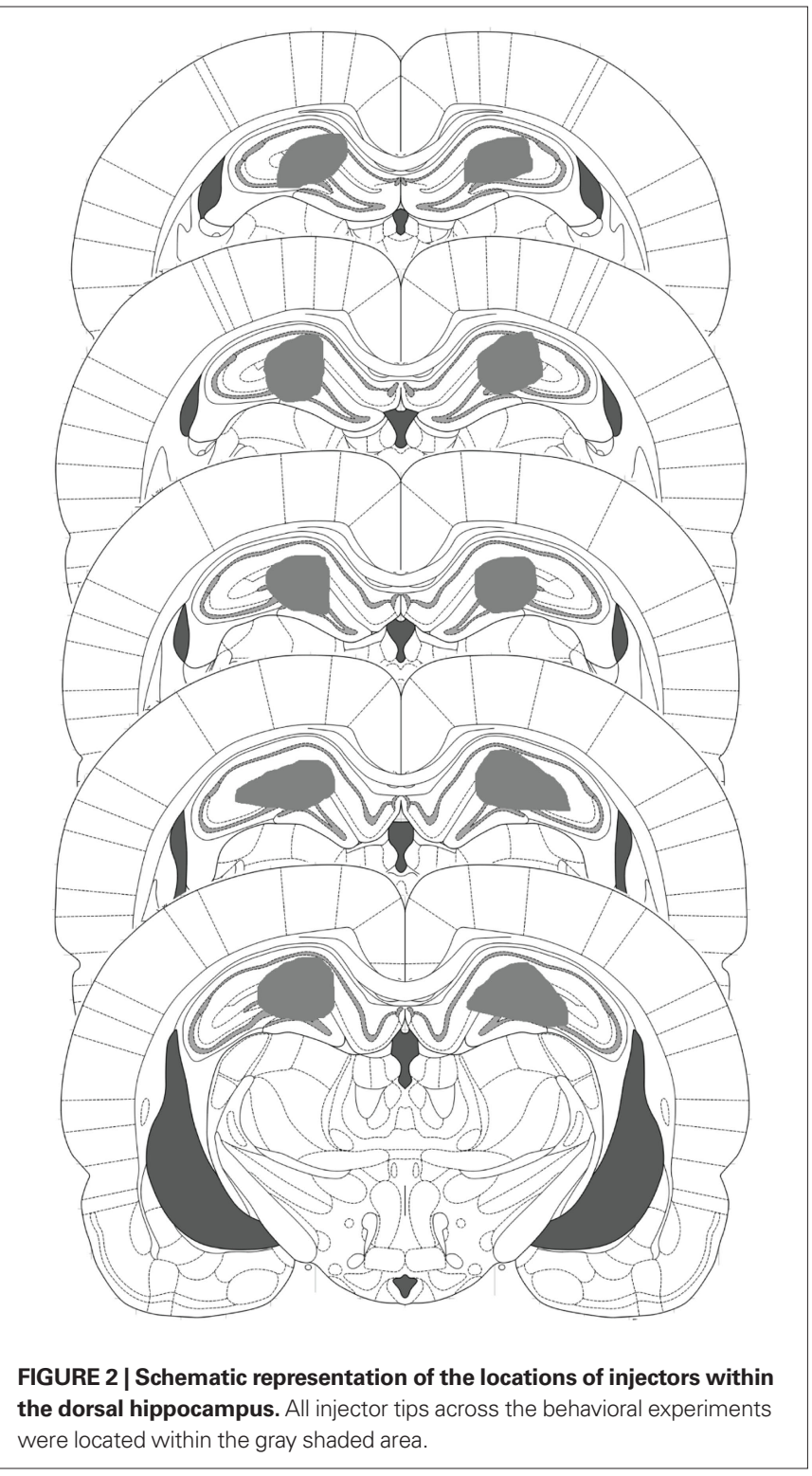

not differing from each other. Thus in the Zif268 ASO-infused rats, the 5-min context fear test would not be expected to provide sufficient context exposure to facilitate contextual freezing.

\section{The disruptive effect of Zif268 requires memory updating conditions}

In order to replicate the previous findings of Biedenkapp and Rudy, and to establish whether the presence of the footshock is necessary to destabilize the previously learned context memory, rats were infused with Zif268 ASO/MSO prior to a 2-min context re-exposure session (Figure 5A). Zif268 ASO infusion did not appear to impair the reconsolidation of the retrieved contextual memory, as both groups subsequently conditioned to the immediate shock to a similar degree $\left(F_{(1,10)}=1.80, p=0.209\right)$. There was, however, a suggestion that the Zif268 ASO-infused group froze at a lower level than controls. Coupled with the previous finding that context re-exposure does upregulate Zif268 protein expression, a subsequent group of rats were given weaker pre-exposure to the context

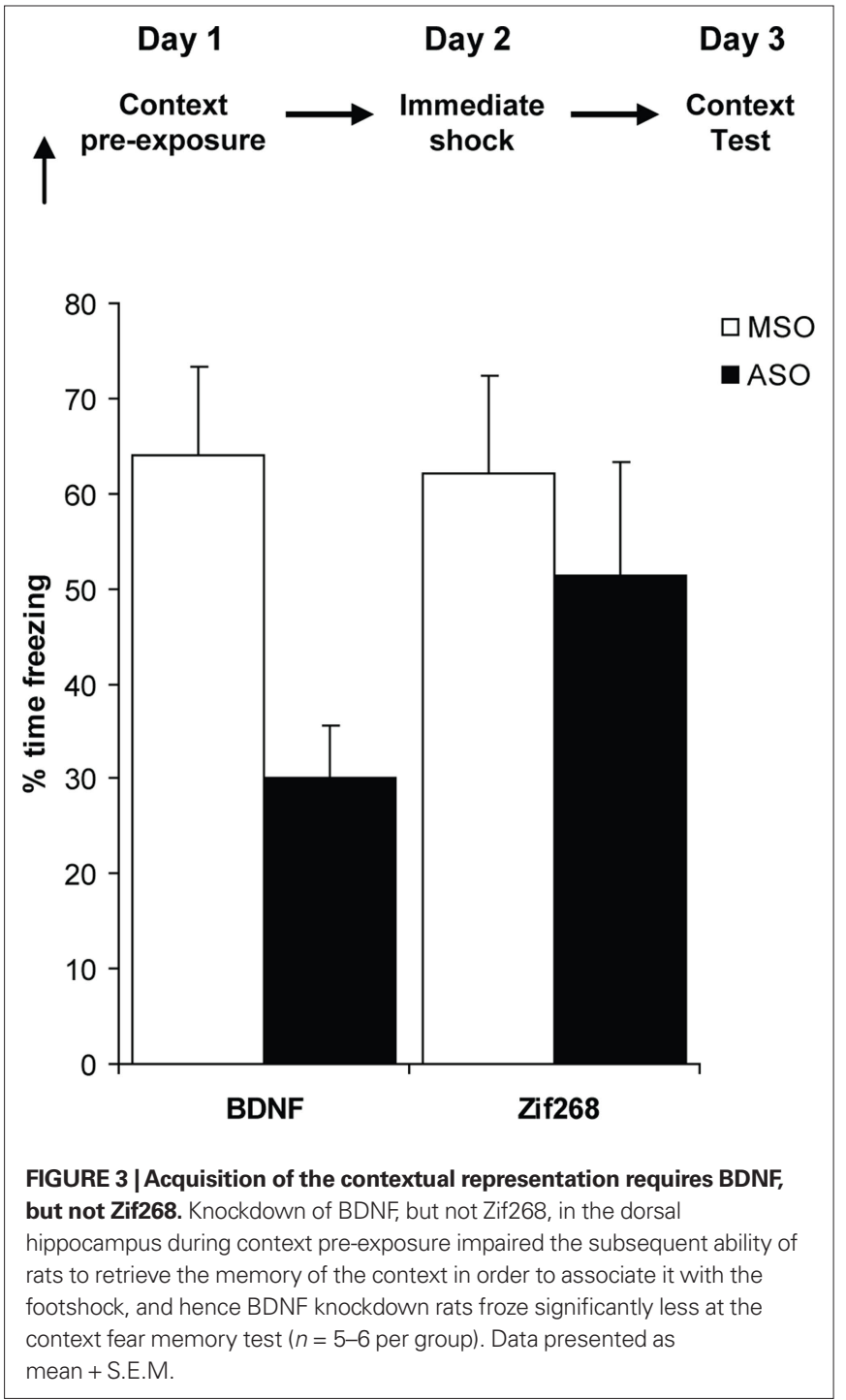

(Figure 5B). Under these conditions, Zif268 ASO infusion prior to a 2-min context re-exposure did impair the reconsolidation of the retrieved contextual memory $\left(F_{(1,10)}=5.21, p=0.046\right)$. Therefore, weaker context exposure results in a contextual memory that is more vulnerable to reconsolidation impairment.

It was not simply the presence of the footshock during the immediate shock session, but the memory updating triggered by the footshock, that engaged memory reconsolidation (Figure 5C). In rats that were given footshock at the end of each context exposure during the pre-exposure session, infusion of Zif268 ASO during a subsequent immediate shock session did not affect later contextual freezing $\left(F_{(1,9)}=1.13, p=0.315\right)$. Thus an already strong contextual fear memory does not appear to undergo reconsolidation when retrieved by an immediate shock session.

\section{DISCUSSION}

The present results demonstrate that the conditioning of fear to a retrieved contextual memory within a CPFE paradigm engages and requires reconsolidation-specific cellular mechanisms within the dorsal hippocampus. Specifically, following context pre-exposure, 


\section{A}
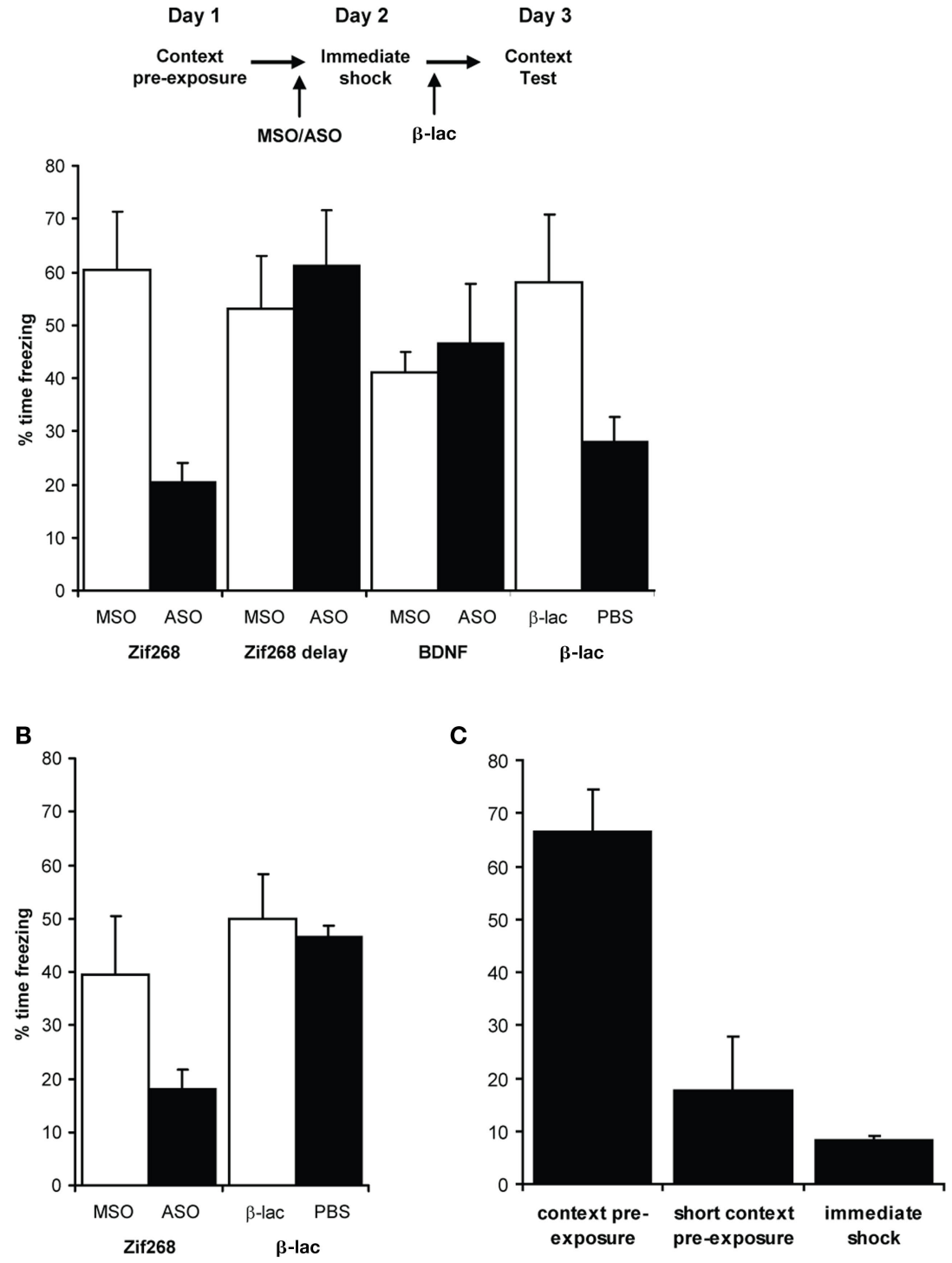

FIGURE 4 | Updating the contextual representation requires memory destabilization and reconsolidation. (A) Rats were pre-exposed to the context, and then infused into the dorsal hippocampus at the time of a subsequent immediate shock, with the updated memory assessed in a contextual fear test. Whereas knockdown of BDNF had no effect, knockdown of Zif268 and inhibition of synaptic protein degradation ( $\beta$-lac) both significantly impaired contextual freezing. Zif268 knockdown no longer had an effect when delayed by $4 \mathrm{~h}$ after the immediate shock session ( $n=5-6$ per group).

(B) Retraining of the impaired rats. The groups showing an impairment were retrained the next week with further immediate shock and context fear test immediate shock upregulated the expression of Zif268 protein in the dorsal hippocampus, the antisense oligodeoxynucleotidemediated knockdown of which prevented subsequent contextual freezing. In contrast, it was the knockdown of BDNF that was necessary to impair the initial formation of the contextual representation. Therefore, there are doubly dissociable hippocampal cellular mechanisms of context memory consolidation and fear conditioning to the retrieved memory that match the specific mechanisms of hippocampal contextual fear memory consolidation and reconsolidation (Lee et al., 2004). Moreover, given the critical engagement of reconsolidation-specific plasticity mechanisms in the dorsal hippocampus following the immediate shock phase of the procedure, the current data indicate both that the dorsal hippocampus contributes to context-footshock processing, and that the reconsolidation process is functionally recruited to update the neutral contextual representation to a contextual fear memory. 


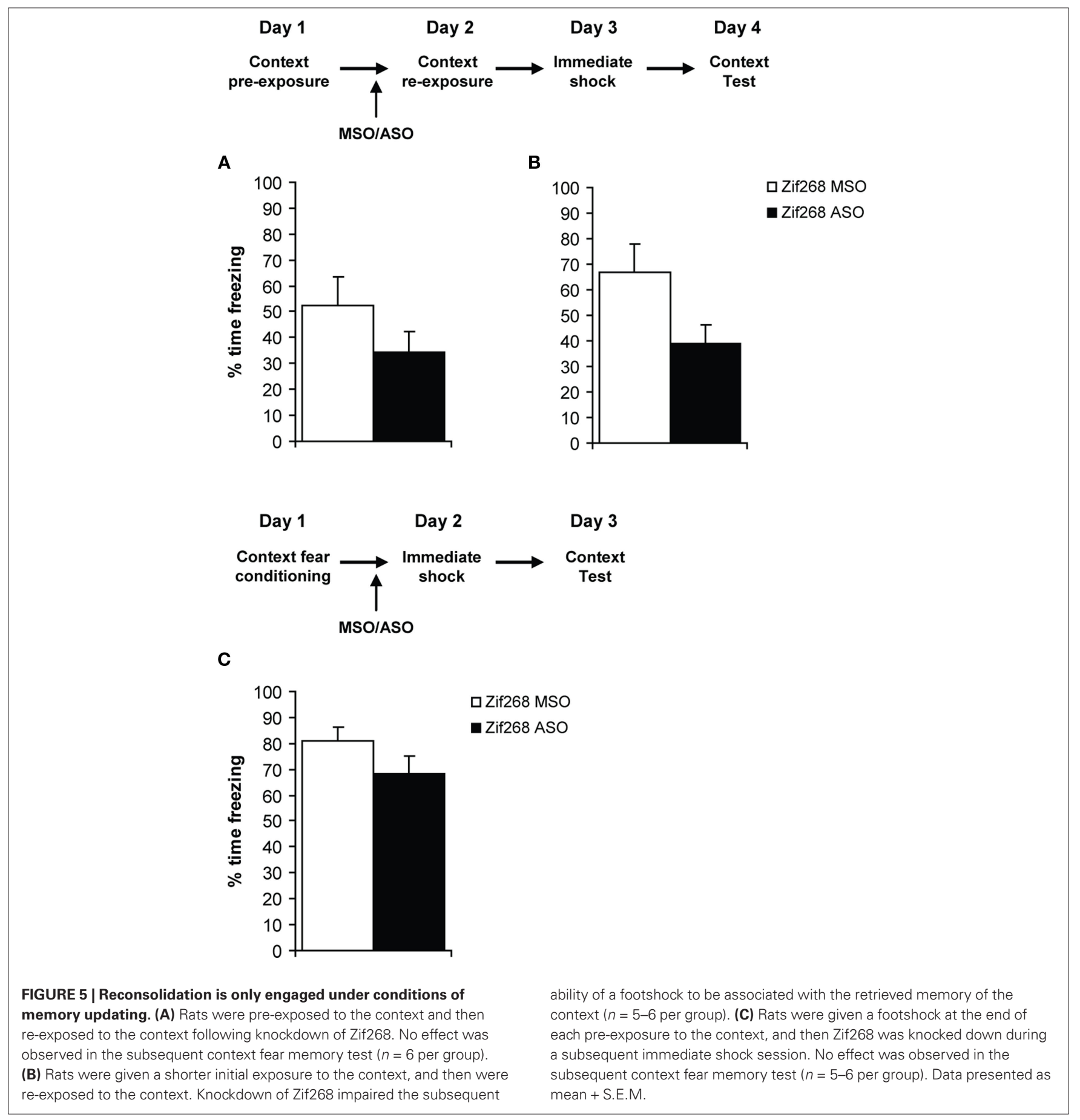

The CPFE paradigm employed here has been extensively studied as a procedure to isolate the learning of contextual representations in the absence of emotionally-salient content (Fanselow, 2000; Biedenkapp and Rudy, 2004; Matus-Amat et al., 2004, 2007). The integrity of the contextual representation is assessed through the ability to associate its retrieved memory with footshock, through the immediate shock phase, and thereby subsequently support conditioned freezing to the context. Inhibition of hippocampal memory retrieval processes at the time of the immediate shock impairs subsequent contextual freezing (Matus-Amat et al., 2004),

indicative that retrieval of the contextual representation is a critical element of the procedure. It has been assumed that the contribution of the hippocampus to both this procedure and contextual fear conditioning is limited to the formation of the contextual representation (Rudy and O'Reilly, 1999; Fanselow, 2000; Maren, 2001), which is subsequently associated with the footshock in the amygdala (LeDoux, 2000; Matus-Amat et al., 2007). However, some evidence suggests that the dorsal hippocampus may play a greater role in fear-related contextual memory processing. Lesions of the entire hippocampus disrupted the reinstatement of extinguished 
contextual fear, indicative of a context-shock association being stored within the hippocampus (Frohardt et al., 2000). Moreover, in the CPFE paradigm, infusion of lidocaine into the dorsal hippocampus immediately after the immediate shock session produced a profound deficit in subsequent contextual freezing (Chang et al., 2008). Given the post-trial nature of the intervention, this finding is inconsistent with the hippocampus only being necessary to retrieve the contextual representation, and is instead consistent with a role for the dorsal hippocampus in context-shock processing.

Gene expression studies also point toward dorsal hippocampal involvement in context-shock processing. It is consistently observed that the upregulation of immediate-early genes in the dorsal hippocampus is most pronounced under conditions of context-shock learning (Impey et al., 1998; Tischmeyer and Grimm, 1999; Hall et al., 2000; von Hertzen and Giese, 2005, but see Huff et al., 2006). Moreover, the present data indicate that dorsal hippocampal BDNF protein levels are significantly increased following exposure to a novel environment alone, in the absence of any fear conditioning, suggesting that BDNF-mediated hippocampal plasticity contributes to the encoding of a contextual representation. The BDNF-regulated protein Arc is also upregulated in the dorsal hippocampus following contextual fear conditioning (Lee et al., 2004), and so the precise mnemonic contribution of the dorsal hippocampus to contextual fear conditioning remains unclear. The dorsal hippocampus is certainly capable of supporting contextual and spatial representations. However, the enhanced hippocampal gene expression under conditions of contextual fear conditioning is equally consistent with both a stronger contextual representation and a contribution to context-shock processing. Nevertheless, the present data reveal that the cellular mechanisms of context representation formation (BDNF) and footshock updating of that representation (Zif268) are doubly dissociable.

Doubly dissociable mechanisms of memory consolidation and reconsolidation have previously been capitalized upon to isolate the relative contributions of the two processes to memory updating. In an inhibitory avoidance procedure, memory consolidation depends specifically upon the expression of the immediate-early gene $C$ / $E B P \beta$ in the dorsal hippocampus (Taubenfeld et al., 2001), whereas memory reconsolidation selectively requires $C / E B P \beta$ expression in the amygdala (Milekic et al., 2007). In a second-order conditioning adaptation of inhibitory avoidance learning, it was hippocampal $C / E B P \beta$ expression that was necessary to acquire the second-order relationship (Tronel et al., 2005). Therefore, consolidation mechanisms are engaged when new information is linked to a retrieved memory. This is not unexpected, when one considers that secondorder memories are mediated either by the formation of a new second order stimulus-first order stimulus association, or a new association between the second-order stimulus and the retrieved representation of the outcome (Gewirtz and Davis, 2000). As such, the second-order responding does not depend upon the updating of the original first-order memory. In contrast, the present selective and functional engagement of reconsolidation mechanisms when fear is conditioned to the retrieved memory of the context is consistent with the contextual representation being modified to incorporate the salient footshock information. While the basolateral amygdala is certainly involved in context-shock plasticity (Phillips and LeDoux, 1992; Maren et al., 1996; Matus-Amat et al., 2007), this does not preclude an additional functional contribution from hippocampal structures, at least within the CPFE setting (Chang et al., 2008).

The interpretation that reconsolidation mediates the modification of hippocampal memory content is further supported by the comparison of the effects of Zif268 knockdown and proteasome inhibition upon contextual freezing after retraining. If hippocampal Zif268 knockdown simply prevented the conditioning of fear to the retrieved memory of the context, rather than impairing memory modification per se, then the contextual representation itself should have remained intact. However, subsequent retraining with an immediate shock in the absence of any treatment revealed a persistent deficit in contextual freezing, suggesting instead that the contextual representation had previously been disrupted. This indicates first that Zif268 knockdown impaired the reconsolidation of the contextual representation, which is consistent with previous data (Lee et al., 2004), and hence that reconsolidation supports the modification of the contextual memory. Thus the existing contextual memory is destabilized in order to incorporate the footshock information during reconsolidation, and interruption of the reconsolidation process by Zif268 knockdown both prevents the fear conditioning, and impairs the original contextual representation. This account is supported by the contrasting effect of inhibition of synaptic protein degradation by $\beta$-lac. $\beta$-lac has previously been shown to prevent the destabilization of a retrieved contextual fear memory (Lee et al., 2008), and here impaired initial contextual freezing, but had no effect on the CPFE elicited during retraining. Without destabilization of the contextual representation, the new footshock information cannot be incorporated into the contextual representation during the reconsolidation process, and so little contextual freezing was observed in the first test. However, the context memory itself remains intact and so is capable of supporting the CPFE when subsequently retrained in the absence of any treatment. Chang et al. (2008) had previously shown a similar pattern of results to that of $\beta$-lac when using intra-dorsal hippocampal infusions of lidocaine. This may be attributed to the neuronal inhibitory effects of lidocaine, which are consistent with a failure to destabilize the hippocampal memory, given that memory destabilization also appears to depend upon signal transduction at various cell surface receptors and ion channels (Ben Mamou et al., 2006; Suzuki et al., 2008).

Hippocampal memory reconsolidation has previously been linked to the strengthening of contextual fear memories (Lee, 2008). The present data suggest that this involvement in memory strengthening can be extended to neutral contextual memories. Using the standard context pre-exposure parameters of $500 \mathrm{~s}$ over six episodes, there was little evidence for a context memory reconsolidation deficit when Zif268 was knocked down during a 2-min context re-exposure session. This is consistent with previous data from Biedenkapp and Rudy (2004), who showed similar findings with both a 5-s and a 1-min context re-exposure. However, the 2-min context re-exposure here upregulated dorsal hippocampal Zif268 expression, albeit to a significantly lesser extend than did the immediate shock. This was somewhat consistent with a previous study of JunB expression (Strekalova et al., 2003), and was suggestive that context re-exposure does engage memory reconsolidation. Indeed, when a shorter context pre-exposure session (380 s over 
three episodes) was used, re-exposure-elicited reconsolidation was demonstrated by a Zif268 knockdown-mediated impairment. Thus, the shorter initial training may have resulted in a weaker contextual representation that was strengthened, using reconsolidation mechanisms, by the re-exposure session. Indeed, it might be predicted that under conditions of shorter initial training, re-exposure to the context may result in a similar level of Zif268 upregulation as was observed in the rats exposed to immediate shock following the extended context pre-exposure. In contrast, the normal preexposure may have been sufficient to result in asymptotic levels of context representation acquisition (Lee, 2009). While this interpretation is consistent with prior data using intra-hippocampal protein synthesis inhibition in the spatial water maze (RodriguezOrtiz et al., 2008), as well as other demonstrations that well-learned memories do not undergo reconsolidation unless in the presence of new information (Rodriguez-Ortiz et al., 2005; Morris et al., 2006; Winters et al., 2009), it is somewhat undermined by the observation that freezing levels in missense-infused controls were lower with the longer context pre-exposure. It remains possible that such a difference may be attributable to differing levels of fear expression across batches of rats, which obscured the strength of the memory itself. However, further studies will be necessary conclusively to demonstrate that there is a threshold of context memory learning that influences the subsequent capacity for context re-exposure to trigger memory reconsolidation.

One counter-argument to the functional involvement of reconsolidation in memory updating is that it is merely the presence of a salient event during the immediate shock session that triggers reconsolidation of the contextual representation. Thus the presence of the footshock during the immediate shock session destabilizes the contextual memory not because of any memory modification induced, but simply due to its emotional salience. However, immediate shock was not able to destabilize a

\section{REFERENCES}

Ben Mamou, C., Gamache, K., and Nader, K. (2006). NMDA receptors are critical for unleashing consolidated auditory fear memories. Nat. Neurosci. 9 , 1237-1239.

Biedenkapp, J. C., and Rudy, J. W. (2004). Context memories and reactivation: constraints on the reconsolidation hypothesis. Behav. Neurosci. 118, 956-964.

Chang, S.-D., Chen, D.-Y., and Liang, K. C. (2008). Infusion of lidocaine into the dorsal hippocampus before or after the shock training phase impaired conditioned freezing in a two-phase training task of contextual fear conditioning. Neurobiol. Learn. Mem. 89, 95-105.

Dudai, Y. (2006). Reconsolidation: the advantage of being refocused. Curr. Opin. Neurobiol. 16, 174-178.

Fanselow, M. S. (2000). Contextual fear, gestalt memories, and the hippocampus. Behav. Brain Res. 110, 73-81.

Fendt, M., and Fanselow, M.S. (1999). The neuroanatomical and neurochemical basis of conditioned fear. Neurosci. Biobehav. Rev. 23, 743-760.

Forcato, C., Rodriguez, M. L., Pedreira, M. E., and Maldonado H. (2010) Reconsolidation in humans opens up declarative memory to the entrance of new information. Neurobiol. Learn. Mem. 93, 77-84.

Frohardt, R. J., Guarraci, F.A., and Bouton, M.E. (2000). The effects of neurotoxic hippocampal lesions on two effects of context after fear extinction. Behav. Neurosci. 114, 227-240.

Gewirtz, J. C., and Davis, M. (2000). Using pavlovian higher-order conditioning paradigms to investigate the neural substrates of emotional learning and memory. Learn. Mem. 7, 257-266.

Hall, J., Thomas, K. L., and Everitt, B. J. (2000). Rapid and selective induction of BDNF expression in the hippocampus during contextual learning. Nat. Neurosci. 3, 533-535.

Huff, N.C., Frank, M., Wright-Hardesty, K., Sprunger, D., Matus-Amat, P., Higgins, E., and Rudy, J. W. (2006). Amygdala strong contextual fear memory, formed previously by delivering a footshock at the end of each episode of context pre-exposure. Thus the capacity of the immediate shock to destabilize the contextual memory in the present study is likely due to the new footshock information present over and above the already-learned contextual representation. This interpretation is in accordance with the findings of Winters et al. (2009), who demonstrated in an object recognition paradigm that new information was required in order to destabilize strong, but not weak, memories. Moreover, the previous observation of context-shock pairings being sufficient to destabilize an existing contextual fear memory (Lee, 2008) was likely facilitated by the relatively weak level of initial contextual fear conditioning. In the previous study, initial conditioning led to approximately $50 \%$ time spent freezing, rising to $60 \%$ with further learning. In contrast, the final levels of contextual freezing after shocked pre-exposure were closer to $80 \%$. This is again consistent with the requirement for the memory reactivation session, here the immediate shock session, to engage further learning due to incomplete previous learning (RodriguezOrtiz et al., 2005, 2008; Lee, 2009).

In summary, the present data demonstrate that in order to update a neutral contextual representation to a contextual fear memory, the contextual representation is first destabilized and then reconsolidated incorporating the emotionally salient information. Along with the further support for reconsolidation being functionally relevant for the strengthening of memories, this places the reconsolidation process in a central role for memory modification, at least for hippocampal memories. It remains to be determined the mechanisms by which the reconsolidation process is constrained by the necessity for memory updating conditions.

\section{ACKNOWLEDGMENTS}

I thank Charlotte Flavell and Dave Barber for technical assistance. regulation of immediate-early gene expression in the hippocampus induced by contextual fear conditioning. J. Neurosci. 26, 1616-1623.

Hupbach, A., Gomez, R., Hardt, O., and Nadel, L. (2007). Reconsolidation of episodic memories: a subtle reminder triggers integration of new information. Learn. Mem. 14, 47-53.

Impey, S., Smith, D. M., Obrietan, K., Donahue, R., Wade, C., and Storm, D. R. (1998). Stimulation of cAMP response element (CRE)-mediated transcription during contextual learning. Nat. Neurosci. 1, 595-601.

LeDoux, J. E. (2000). Emotion circuits in the brain. Annu. Rev. Neurosci. 23 , 155-184.

Lee, J. L. C. (2008). Memory reconsolidation mediates the strengthening of memories by additional learning. Nat. Neurosci. 11, 1264-1266.

Lee, J. L. C. (2009). Reconsolidation: maintaining memory relevance. Trends Neurosci. 32, 413-420.

Lee, J. L. C., Everitt, B. J., and Thomas, K. L. (2004). Independent cellular processes for hippocampal memory consolidation and reconsolidation. Science 304, 839-843.

Lee, S. H., Choi, J. H., Lee, N., Lee, H. R., Kim, J. I., Yu, N. K., Choi, S. L., Lee, S. H., Kim, H., and Kaang, B. K. (2008). Synaptic protein degradation underlies destabilization of retrieved fear memory. Science 319, 1253-1256.

Maren, S. (2001). Neurobiology of Pavlovian fear conditioning. Annu. Rev. Neurosci. 24, 897-931.

Maren, S., Aharonov, G., Stote, D. L., and Fanselow, M. S. (1996). N-methyl-Daspartate receptors in the basolateral amygdala are required for both acquisition and expression of conditional fear in rats. Behav. Neurosci. 110, 1365-1374.

Matus-Amat, P., Higgins, E. A., Barrientos, R. M., and Rudy, J.W. (2004). The role of the dorsal hippocampus in the acquisition and retrieval of context memory representations. J. Neurosci. 24, 2431-2439.

Matus-Amat, P., Higgins, E. A., Sprunger, D., Wright-Hardesty, K., and Rudy, J. 
W. (2007). The role of dorsal hippocampus and basolateral amygdala NMDA receptors in the acquisition and retrieval of context and contextual fear memories. Behav. Neurosci. 121, 721-731.

Milekic, M.H., Pollonini, G., and Alberini, C. M. (2007). Temporal requirement of C/EBPbeta in the amygdala following reactivation but not acquisition of inhibitory avoidance. Learn. Mem. 14, 504-511.

Morris, R. G., Inglis, J., Ainge, J.A., Olverman, H. J., Tulloch, J., Dudai, Y., and Kelly P. A. (2006). Memory reconsolidation: sensitivity of spatial memory to inhibition of protein synthesis in dorsal hippocampus during encoding and retrieval. Neuron 50, 479-489.

Nader, K., and Hardt, O. (2009). A single standard for memory: the case for reconsolidation. Nat. Rev. Neurosci. 10, 224-234.

Phillips, R. G., and LeDoux, J. E. (1992). Differential contribution of amygdala and hippocampus to cued and contextual fear conditioning. Behav. Neurosci. 106, 274-285.

Rodriguez-Ortiz, C. J., De la Cruz, V., Gutierrez, R., and Bermudez-Rattoni, F. (2005). Protein synthesis underlies post-retrieval memory consolidation to a restricted degree only when updated information is obtained. Learn. Mem. 12, 533-537.

Rodriguez-Ortiz, C. J., Garcia-DeLaTorre, P., Benavidez, E., Ballesteros, M. A., and Bermudez-Rattoni, F. (2008). Intrahippocampal anisomycin infusions disrupt previously consolidated spatial memory only when memory is updated. Neurobiol. Learn. Mem. 89, 352-359.

Rudy,J.W., Barrientos, R. M., and O’Reilly, R.C. (2002). Hippocampal formation supports conditioning to memory of a context. Behav. Neurosci. 116, 530-538.

Rudy, J. W., and O'Reilly, R. C. (1999). Contextual fear conditioning, conjunctive representations, pattern completion, and the hippocampus. Behav. Neurosci. 113, 867-880.

Sara, S. J. (2000). Retrieval and reconsolidation: toward a neurobiology of remembering. Learn. Mem. 7, 73-84.

Strekalova, T., Zorner, B., Zacher, C., Sadovska, G., Herdegen, T., and Gass, P. (2003). Memory retrieval after contextual fear conditioning induces c-Fos and JunB expression in CA1 hippocampus. Genes Brain Behav. 2, 3-10.

Suzuki, A., Mukawa, T., Tsukagoshi, A., Frankland, P.W., and Kida, S. (2008). Activation of LVGCCs and CB1 receptors required for destabilization of reactivated contextual fear memories. Learn. Mem. 15, 426-433.

Taubenfeld, S. M., Milekic, M. H., Monti, B., and Alberini, C. M. (2001). The consolidation of new but not reactivated memory requires hippocampal C/EBP beta. Nat. Neurosci. 4, 813-818.

Tischmeyer, W., and Grimm, R. (1999). Activation of immediate early genes and memory formation. Cell. Mol. Life Sci. 55, 564-574.

Tronel, S., Milekic, M. H., and Alberini, C. M. (2005). Linking new information to a reactivated memory requires consolidation and not reconsolidation mechanisms. PLoS Biol. 3, e293. doi: 10.1371/journal.pbio.0030293.

Tronson, N. C., and Taylor, J. R. (2007) Molecular mechanisms of memory reconsolidation. Nat. Rev. Neurosci. 8, 262-275.

von Hertzen, L. S., and Giese, K. P. (2005). Memory reconsolidation engages only a subset of immediate-early genes induced during consolidation. J. Neurosci. 25, 1935-1942.

Winters, B. D., Tucci, M. C., and DaCosta-Furtado, M. (2009). Older and stronger object memories are selectively destabilized by reactivation in the presence of new information. Learn. Mem. 16, 545-553.
Ying, S. W., Futter, M., Rosenblum, K., Webber, M. J., Hunt, S. P., Bliss, T. V., and Bramham, C. R. (2002). Brainderived neurotrophic factor induces long-term potentiation in intact adult hippocampus: requirement for ERK activation coupled to CREB and upregulation of Arc synthesis. J. Neurosci. 22, 1532-1540.

Conflict of Interest Statement: The author declares that the research was conducted in the absence of any commercial or financial relationships that could be construed as a potential conflict of interest.

Received: 05 May 2010; paper pending published: 15 September 2010; accepted: 06 October 2010; published online: 11 November 2010.

Citation: Lee JLC (2010) Memory reconsolidation mediates the updating of hippocampal memory content. Front. Behav. Neurosci. 4:168. doi: 10.3389/ fnbeh.2010.00168

Copyright (c) 2010 Lee. This is an openaccess article subject to an exclusive license agreement between the authors and the Frontiers Research Foundation, which permits unrestricted use, distribution, and reproduction in any medium, provided the original authors and source are credited. 